\title{
Local Scale Groundwater Vulnerability Assessment Using GIS-Based DRASTIC Model, Emphasis on Groundwater Quality
}

\author{
Yongxiang ZHANG ${ }^{\text {a }}$, Ruitao JIA ${ }^{\text {a }}$, Jin WU ${ }^{\mathrm{a}, 1}$, Huaqing $\mathrm{WANG}^{\text {a,b }}$ and Zhuoran $\mathrm{LUO}^{\mathrm{a}}$ \\ ${ }^{a}$ Faculty of Architecture, Civil and Transportation Engineering, Beijing University of \\ Technology, Beijing 100124, China \\ ${ }^{\mathrm{b}}$ Normandie UNIV, UNIHAVRE, UMR 6294, CNRS, LOMC, 76600 Le Havre, France
}

\begin{abstract}
Groundwater vulnerability assessment is a basic work for groundwater exploitation and protection. The Chaoyang district of Beijing was selected and investigated in this study. Groundwater vulnerability index system in Chaoyang district was constructed based on hydrogeological settings of local region, the human influence and the DRASTIC model. The comprehensive vulnerability assessment was carried out with weights of 0.4 and 0.6 for the intrinsic vulnerability and the specific vulnerability, respectively. In this study, total 9 hydrogeological parameters were considered, and the diagram of groundwater vulnerability assessment results in Chaoyang District was obtained by using DRASTIC index and overlay weighted method. The groundwater quality is poor in the southwest of Chaoyang District. The correlation analysis between total hardness, total dissolved solids and vulnerability results was carried out, and the correlation results were 06 and 0.7 , respectively. The area with high groundwater vulnerability is also the regions with serious groundwater pollution, indicating that the assessment results are objective and reasonable, which can provide prevention and control of groundwater reference for the management department in the future, so as to reduce the risk of pollution.
\end{abstract}

Keywords. DRASTIC, geographic information system, groundwater quality, TH and TDS concentration, Chaoyang district

\section{Introduction}

As one of the material bases for the existence of earth organisms, groundwater is facing the great pressure brought by the rapid development of urbanization. This water is mainly used for drinking and industrial and agricultural development have affected the quality of groundwater around the world. [1]. Therefore, groundwater pollution vulnerability evaluation is an significant task for groundwater resources management and protection [2]. In recent years, Groundwater vulnerability assessment is of great significance to the rational development, utilization and protection of groundwater and the sustainable development of social economy. [3].

DRASTIC is the most widely used in groundwater vulnerability assessment, this method has strong subjectivity in obtaining and assigning evaluation parameters [4]. In

\footnotetext{
1 Jin Wu, Faculty of Architecture, Civil and Transportation Engineering, Beijing University of Technology, Beijing 100124, China; E-mail: Wujin@bjut.edu.cn.
} 
this study, 9 hydrogeological parameters were used to evaluate groundwater vulnerability. Groundwater vulnerability assessment and visualization map can be used for crucial prediction tools for decision-makers in restoring groundwater quality.

Groundwater vulnerability is the complexity of surface and underground conditions that affect the entry of pollutants into aquifers [5]. Some studies choose a larger regional scale, but less depiction of small scales such as Chaoyang District, Beijing. At present, some scholars evaluate groundwater vulnerability from multiple angles, methods and means, and the evaluation model is becoming more and more mature [6]. This study uses GIS technology and DRASTIC method has been used to obtain groundwater potential area map and identify vulnerable areas. The collected data are processed and statistically analyzed by ArcGIS, and then the groundwater vulnerability index is obtained by grid algebraic operation, and finally the groundwater vulnerability evaluation results are obtained [7].

This study was conducted in the Chaoyang district of Beijing in the capital of China to evaluate groundwater vulnerability and verify risk sensitive areas. The total hardness (TH) and total dissolved solids (TDS) are significant index in groundwater.

In this study, the DRASTIC model is used to evaluate the groundwater vulnerability in Chaoyang District. Considering the impact of water quality, the quality of groundwater quality can be reflected to a certain extent through the correlation analysis of water quality parameters such as TDS and TH. The research results can provide strong technical support for the prevention and control of groundwater pollution in Chaoyang District.

\section{Materials and Methods}

\subsection{Study Area}

Chaoyang district with a population of about 3.473 million people, is a district located in east Beijing, the capital of China, the specific geographic location is shown in figure (a). It bounded approximately by longitudes $116^{\circ} 21^{\prime}-116^{\circ} 42^{\prime} \mathrm{E}$ and latitudes $39^{\circ} 48^{\prime}-$ $40^{\circ} 09^{\prime} \mathrm{N}$, covering an area of $470.8 \mathrm{~km}^{2}$, it is the largest district in the center of Beijing, with an average altitude of $34 \mathrm{~m}$, the terrain inclines slowly from northwest to southeast, and the slope is generally between $1 / 1000$ and $1 / 2500$ [8]. Chaoyang district belongs to the continental type and semi-arid monsoon climate, with annual precipitation of $581 \mathrm{~mm}$, summer precipitation accounts for $75 \%$ of the whole year. As shown in figure 1(b), the groundwater in this area flows from northwest to southeast. There are three groundwater depression cones, which are Jiuxianqiao, Hongmiao and Dajiaoting. The regional aquifer is mainly composed of Quaternary loose sediments, gradually thickening from west to East, Dongba, Jinzhan area more than $450 \mathrm{~m}$.

As a rich natural resource, groundwater has been exploited and utilized in large quantities, which leads to the problem of groundwater environmental pollution and damage increasingly serious. Groundwater pollution mainly comes from the impact of human, agricultural and industrial activities. With the optimization of industrial structure and the change of land use pattern in Chaoyang District, the distribution of groundwater vulnerability has changed greatly. 

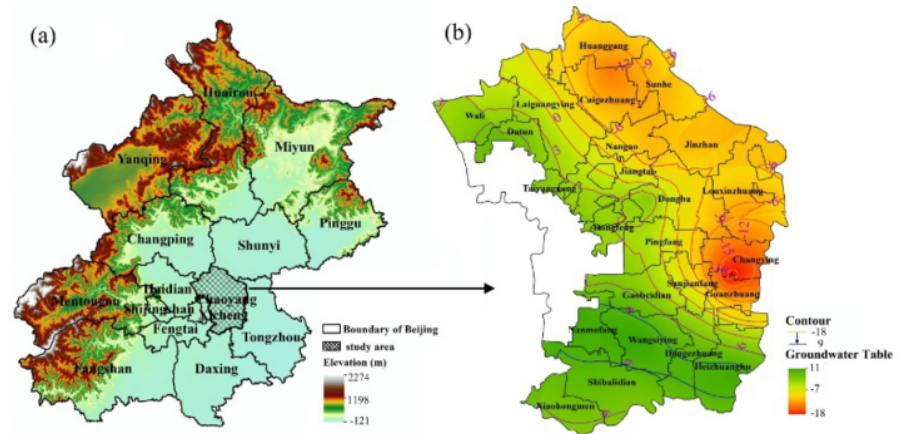

Figure 1. (a) Schematic diagram of the location; (b) Groundwater flow field map of the study area.

\subsection{Data Sources and Framework}

The vulnerability score is between 1 and 10 . The greater the value, the higher the vulnerability level. See Table 1 and table 2 for the scope and score values of inherent vulnerability and unique vulnerability indicators, respectively. Hydrogeological data are from 37 boreholes in the region, groundwater quality data come from the monitoring values of monitoring wells in Chaoyang District for many years, other data are from Beijing water resources bulletin and Chaoyang District statistical yearbook in 2018. According to the groundwater vulnerability assessment model, the comprehensive groundwater vulnerability index is obtained by overlaying the impact factor score map with ArcGIS software.

Table 1. Groundwater intrinsic vulnerability rating and weighting values.

\begin{tabular}{|c|c|c|c|c|c|}
\hline $\mathrm{D}(\mathrm{w}: 5) / \mathrm{m}$ & $\mathrm{R}(\mathrm{w}: 4) / \mathrm{mm}$ & $\mathrm{A}(\mathrm{w}: 3)$ & S(w:5) & $\mathrm{C}(\mathrm{w}: 3) / \mathrm{m} / \mathrm{d}$ & Rating \\
\hline 30.5 & 0 & clay & clay & 0 & 1 \\
\hline 26.7 & 51 & loam & loam & 4.1 & 2 \\
\hline 22.9 & 71.4 & sand loam & sand loam & 12.2 & 3 \\
\hline 15.2 & 91.8 & mud & mud & 20.3 & 4 \\
\hline 12.1 & 117.2 & mud sand & mud sand & 28.5 & 5 \\
\hline 9.1 & 147.6 & fine sand & fine sand & 34.6 & 6 \\
\hline 6.8 & 178 & medium sand & medium sand & 40.7 & 7 \\
\hline 4.6 & 216 & gritty & gritty & 61.1 & 8 \\
\hline 1.5 & 235 & Sand gravel & Sand gravel & 71.5 & 9 \\
\hline 0 & 254 & Cobble and gravel & Cobble and gravel & 81.5 & 10 \\
\hline
\end{tabular}

Table 2. Groundwater specific vulnerability rating and weighting values.

\begin{tabular}{lllll}
\hline $\mathrm{L}(\mathrm{w}: 5)$ & $\mathrm{Q}(\mathrm{w}: 4)$ & $\mathrm{E}(\mathrm{w}: 4) / \mathrm{m}^{3} / \mathrm{d} \cdot \mathrm{km}^{2}$ & $\mathrm{~F}(\mathrm{w}: 3)$ & Rating \\
\hline Industrial land & $\mathrm{I}$ & $>350$ & $<5$ & 1 \\
land use & II & $250-350$ & $5-15$ & 3 \\
Green land & III & $150-250$ & $15-25$ & 5 \\
Rivers and lakes & IV & $50-150$ & $25-35$ & 7 \\
Cultivated land & V & $<50$ & $>35$ & 10 \\
\hline
\end{tabular}




\subsection{GIS and Constructed Groundwater Vulnerability Index System}

The powerful functions of ArcGIS software, such as data management, data conversion, spatial analysis and grid calculation, have greatly promoted the development of groundwater vulnerability research [9]. The DRASTIC model is jointly developed by USEPA and NWWA. The main hydrogeological parameters are selected as the evaluation index, and the score value of the drastic index is calculated by weighting [4]. Then interpolation method was used for developing the groundwater vulnerability maps(figure 2), including $\mathrm{D}$ (aquifer), $\mathrm{R}$ (recharge), $\mathrm{A}$ (aquifer media), $\mathrm{S}$ (soil texture), $\mathrm{C}$ (hydraulic conductivity), L(land use), Q(groundwater quality), E(mining intensity), $\mathrm{F}$ (fertilizer use) are assigned weight between 1 and 5 [4], and numerical ratings in the range of $1-10$ are given to parameter subclasses according to their perceived contribution to groundwater pollution and vulnerability. Considering the significant impact of human activities on groundwater quality, the intrinsic vulnerability and the specific vulnerability are given the weight of 0.4 and 0.6 respectively for comprehensive vulnerability assessment [10], DRASTIC Index (DI) was determined by the following formula:

$$
\begin{aligned}
& D I=0.4\left(D_{r} D_{w}+R_{r} R_{w}+A_{r} A_{w}+S_{r} S_{w}+C_{r} C_{w}\right)+ \\
& 0.6\left(L_{r} L_{w}+Q_{r} Q_{w}+E_{r} E_{w}+F_{r} F_{w}\right)
\end{aligned}
$$

Where the subscripts $\mathrm{w}$ and $\mathrm{r}$ are the weight and the rating assigned to the respective parameter, respectively.

\subsection{Correlation between Groundwater Vulnerability with Water Quality Parameters}

The results of DRASTIC model were verified by water quality parameters such as TH and TDS in Chaoyang district. The correlation coefficient (R) was used to fit the relationship between water quality parameters and vulnerability. $\mathrm{R}$ was determined by the equation:

$$
R=\frac{n\left(\sum p q\right)-\left(\sum p\right)\left(\sum q\right)}{\sqrt{n \sum p^{2}-\left(\sum p\right)^{2}} \sqrt{n \sum q^{2}-\left(\sum q\right)^{2}}}
$$

Where, $\mathrm{R}$ is correlation coefficient, $\mathrm{n}$ represents the data points, $\mathrm{p}$ and $\mathrm{q}$ are the two variables.
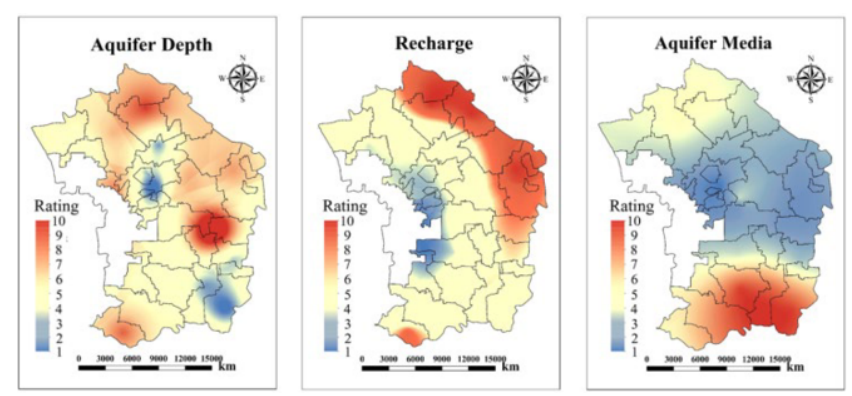

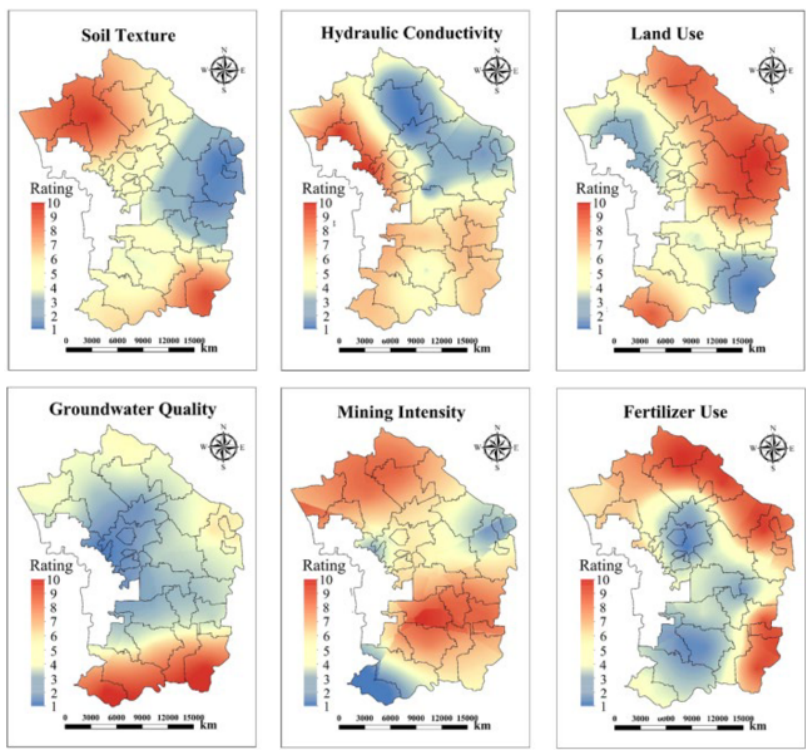

Figure 2. Results of single index evaluation of groundwater vulnerability in 2018 .

\section{Results and Discussion}

\subsection{Groundwater vulnerability distribution}

The comprehensive groundwater vulnerability of Chaoyang district was obtained by superposition of 9 parameters through the DRASTIC model. In the study, DI value varied between 35 and 125, and divided 5 classes: very low (30-60), low (60-80), moderate (80-100), high (100-120), very high (120-150) as shown in figure 3 . The southwest and northeast portion of Chaoyang district show very high DI value covering $24.6 \%$ study area. The northeast of Chaoyang District is mainly cultivated land, the aquifer is shallow, and pollutants are easy to enter groundwater through irrigation. The DI value of study area other regions are shown in table 3.

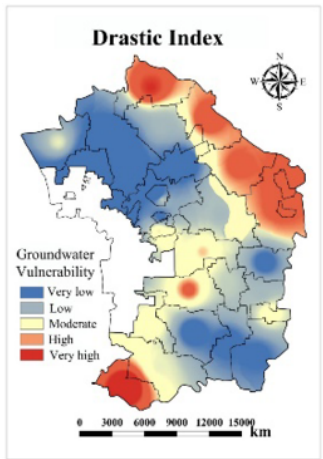

Figure 3. Distribution of groundwater vulnerability in 2018. 
Table 3. Percentage of different ranks of groundwater vulnerability $/ \%$.

\begin{tabular}{llllll}
\hline Vulnerability Classes & very low & low & moderate & high & very high \\
\hline Area (\%) & 24.6 & 17.5 & 22.3 & 19.8 & 15.8 \\
\hline
\end{tabular}

\subsection{Correlation Analysis}

High DI indicates high risk of groundwater pollution, which is verified by TH and TDS values. The correlation value between water quality parameters and $\mathrm{Di}$ is calculated through formula (2), and the R values of Di, th and TDS are 0.6 and 0.7 , respectively which indicates a strong positive correlation (figure 4).

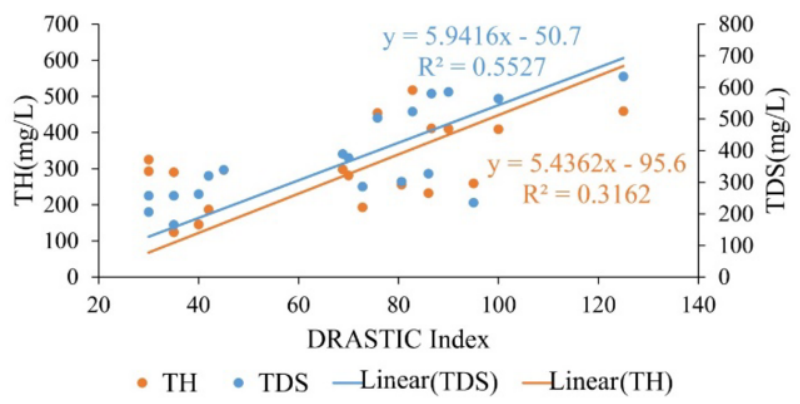

Figure 4. Relationship between the TH, TDS and DRASRIC Index.

\section{Conclusions}

The drastic model and ArcGIS software are combined to evaluate groundwater vulnerability, and the weighted superposition analysis of 9 evaluation factors can intuitively reflect the specific distribution of regional groundwater vulnerability and complete the groundwater vulnerability zoning conveniently and quickly. The high vulnerability are located in the northeast and southwest of Chaoyang district.

The correlation analysis between water quality parameters and vulnerability has a high degree of fitting, which shows that the model has a certain guiding significance for groundwater pollution prevention and groundwater resources management. it is also of great significance to the study of groundwater vulnerability in small-scale regions.

\section{Acknowledgments}

This work was supported by National Key Research and Development Project of China (2016YFC0401404).

\section{References}

[1] Dadgar, Majid Altafi; Nakhaei, Mohammad; Porhemmat, Jahangir. Transient potential groundwater recharge under surface irrigation in semiarid environment: An experimental and numerical study. Hydrological Processes. 2018 Dec; 32(25): 3771-3783. 
[2] Gejl RN, Rygaard M, Henriksen HJ, Rasmussen J and Bjerg PL. Understanding the impacts of groundwater abstraction through long-term trends in water quality. Water Res. 2019 Jun; 156(2): 241251 .

[3] Barzegar R, Moghaddam AA, Deo R, Fijani E, Tziritis E. Mapping groundwater contamination risk of multiple aquifers using multi-model ensemble of machine learning algorithms. Sci. Total Environ. 2018 Apr; 621(11): 697-712.

[4] Aller L, Bennett T, Lehr JH, Petty RJ, Hackett G. Vulnerability and risk evaluation of agricultural nitrogen pollution for Hungary's main aquifer using DRASTIC and GLEAMS models. Journal of Environmental Management. 2009 Jul; 90(10): 2969-2978.

[5] Kazakis N and Voudouris KS. Groundwater vulnerability and pollution risk assessment of porous aquifers to nitrate: modifying the DRASTIC method using quantitative parameters. J. Hydrol. 2015 Jun; 525(3): 13-25.

[6] Ghosh PK, Bandyopadhyay S, Jana NC. Mapping of groundwater potential zones in hard rock terrain using geoinformatics: A case of Kumari watershed in western part of West Bengal Model. Earth Syst. Environ. 2015 Dec; 2(1): 1-12.

[7] Saida S, Tarik H, Abdellah A, Farid H, Hakim B. Developing a fuzzy optimization model for groundwater risk assessment based on improved DRASTIC method. Environmental Earth sciences. 2019 Feb; 78(4): 109-124.

[8] Hao J, Zhang Y, Jia Y, Wang H, Niu C, Gan Y, et al. Assessing groundwater vulnerability and its inconsistency with groundwater quality, based on a modified DRASTIC model: a case study in Chaoyang District of Beijing City. Arabian Journal of Geosciences. 2017 Mar; 10(6):144-159.

[9] Kang J, Zhao L, Li R, Mo H \& Li Y. Groundwater vulnerability assessment based on modified DRASTIC model: a case study in Changli County, China. Geocarto International. 2016 May; 32(7): $749-758$.

[10] Lu Y, He JT, Wang JJ, et al. Uncertainty analysis of groundwater protection and control zoning in Beijing. Plain Environmental Science 2012 Sep; 33(9): 3117-3123. 